\title{
Design of a R-ID in order to determine the position of the vehicle
}

\author{
Frederik Valocký, Peter Drahoš, Oto Haffner, Alena Kozáková \\ Institute of Automotive Mechatronics \\ Slovak University of Technology in Bratislava \\ Bratislava, Slovakia \\ frederik.valocky, peter.drahos, oto.haffner, alena.kozakova [@stuba.sk]
}

\author{
Miloš Orgoň \\ Institute of Multimedia and ICT \\ Slovak University of Technology in Bratislava \\ Bratislava, Slovakia \\ milos.orgon@stuba.sk
}

\begin{abstract}
In this article, we design a road identifier (R-ID). The R-ID must be easily and quickly recognizable by a camera mounted on the vehicle. The camera captures this R-ID and then calculates the position information from it. The article describes the decision-making procedure for the design of an R-ID using camera recognition of geometric shapes. Parameters such as the uniqueness of the R-ID also play a role in this recognition, so that it is not interchangeable with other traffic signs. Another parameter is the percentage needed for the R-ID to be correctly recognized by the camera to obtain the necessary data from the overlay image. The outcome of this article is therefore a road identifier (a pattern) placed on the road which will be captured by the camera mounted on the vehicle .

Index Terms-machine vision, computer vision, autonomous car, data transfer, picture recognition
\end{abstract}

\section{INTRODUCTION}

A UTONOMOUS vehicles are coming to the fore more and more. An important element of the navigation of such a vehicle is the determination of the exact position and thus its location. The most common satellite systems are used to locate the vehicle. However, these satellite systems lose accuracy when vehicles are moving, and of course their reliability is very low in tunnels or covered halls where vehicles are moving. Therefore, it is necessary to devise a method that would be sufficiently accurate and fast enough to ensure the location of the vehicle even in areas where the reliability and possibilities of satellite location is declining [1].

The proposal is to mount a camera in the front of the vehicle, the task of which would be to capture the R-ID road identifier located on the road, and from it to determine the position of the vehicle and the rotation of the vehicle at what angle the vehicle came to this R-ID. These data will be used to enable the vehicle to perform other tasks for autonomous driving with high accuracy.

Recognition of objects and shapes with the camera is much more extensive. The carmakers deal with this problem, where they use the camera to capture traffic signs, which they then display on the display in the vehicle. German motor vehicle manufacturer - Opel equips its selected models with the Opel Eye system This advanced system has four main functions:

The paper was partially supported by the Slovak Research and Development Agency, grant No. APVV-17-0190 and the Slovak Cultural Educational Gran Agency, grant No.038STU-4/2018. traffic sign alert (TSA), lane departure warning (LDW), distance indicator (FDI) and collision warning function front (FCA). Traffic Sign Alert (TSA) works with a new camera that provides a higher detection rate and better features [2]. The camera uses high-frequency image exposure technology and an image processor with significantly higher performance, which allows the system to perform multiple operations simultaneously. In addition to displaying speed limits, the new system also recognizes and displays signs that are related to the maximum speed limit (snow, rain, etc.). The Opel Eye also monitors lane separation lines for lane departure warning (LDW), which is activated immediately if the vehicle leaves the lane without using the turn signals. An audible signal and a mark on the display warns the driver of the possibility of exiting the lane without being signalled by the turn signals. In Fig.1 shows an Opel Eye camera and Fig.2 shows an LDW system [3], [4].

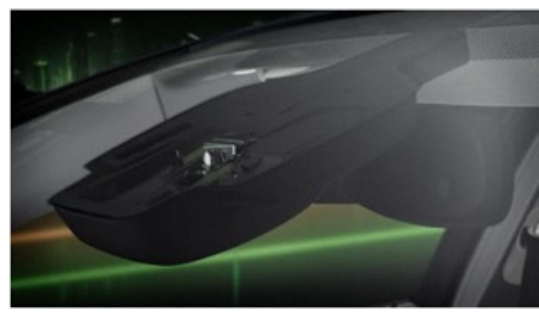

Fig. 1. Innovative Opel Eye camera [3]

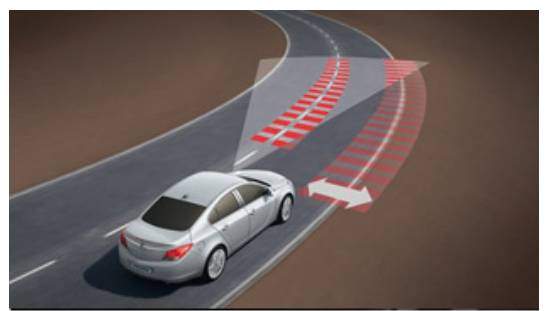

Fig. 2. LDW system in Opel Insignia [4]

In conditions of reduced visibility and at night, a system called "night vision" is very useful. Night vision uses infrared light, which the human eye does not perceive and thus does 
not blind oncoming vehicles. In addition to the usual lights, the road is also illuminated by two infrared headlights and, when the dipped beam headlights are switched on, it expands the driver's field of view by another $150 \mathrm{~m}$. The night visibility system will help identify pedestrians, cyclists, parked vehicles and other obstacles on the road much earlier. An infrared camera is built into the windshield, which records the image of the road ahead and transmits it to the display. The imaging system of a vehicle equipped with "night vision" is shown in Figure 3 [5].

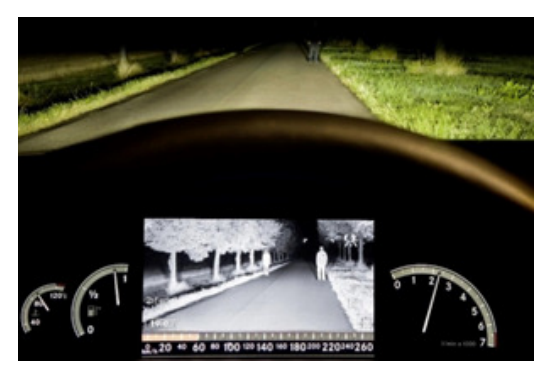

Fig. 3. Display system for a vehicle equipped with "night vision" in a Mercedes-Benz S 550 [6]

\section{IMPORTANCE OF R-ID IN TRANSPORT}

We decided to design the road identifier R ID in order to specify the position of the vehicle in locations where it is not possible to use other sensors than the position sensor etc.. The main task of this identifier is to guide autonomous vehicles in closed production halls where the construction of the building weakens the signals of satellite location systems. The road identifier will be placed on the ground and a camera placed on the vehicle will capture the road identifier. Following the acquisition of the identifier, it calculates the distance between the identifier and the vehicle. It also calculates the angle at which the vehicle approached the identifier and, if it did not meet the requirements of the planned route, would direct the vehicle in the correct direction. Data from the camera will be transmitted to the central unit of the vehicle where they will be processed. The processing of these data from the camera means fusion with other sensors to ensure redundancy and these other sensors are the already mentioned satellite positioning system, vehicle odometry, camera ensuring vehicle safety, vehicle safety means early braking if an obstacle enters the vehicle and this camera subsequently sends a signal that an object has entered the route, which may cause a collision, in which case the vehicle will stop them or perform another necessary action to prevent their collision. Thus, the signal from the COGNEX is7802 camera will be fused with all these sensors .

The accuracy of the road identifier on the road will be ensured by a static satellite signalling sensor, by which the surveyor measures the constant position of the identifier. Since the static satellite locator can determine an accuracy of a few millimetres, each of these patterns will have the exact position determined by such a sensor. Thus, if the vehicle captures the road identifier, it looks at the previous identifier of its position and looks at information from other less accurate sensors, such as the Satellite Location Sensor. If this information fits within the tolerance, the pattern on the ground will calculate the displacement position and thus refine the navigation of the vehicle in an enclosed space [7].

\section{A. Parameters of $R-I D$}

The main task of the tests was to find out which parameters are important precession identifiers. We focused mainly on parameters such as the uniqueness of the identifier, which means that it must not be interchangeable. Identifier recognition and identifier testing was performed using a COGNEX is 7802 camera.

This camera has many pre-programmed functions, such as calibration of the distorted image if the image is at an angle or also pattern search, this function is called PatMax. In our experiments, we mainly used the PatMax function. At the beginning we learned the camera what shape or shape to look in the camera image. And then we moved this shape in different directions and different positions to find out to what extent it can ensure image recognition even when rotated in a vertical position and also what size the identifier can change. to be recognizable below $100 \mathrm{~ms}$.

\section{DAta Transfer Between CAMERA AND Computer}

A camera from COGNEX was chosen for distance measurement, as it has high-quality and widely usable software with a fast support and a friendly In-Sight Explorer configuration environment installed on a computer in which the camera is also controlled. The type of camera used is a monochrome IS7802. This camera has a resolution of $1600 \times 1200$ pixels and the frame rate is 53. As camera lens was used autofocus module with $8 \mathrm{~mm}$ lens (ISAF-7000-8mm) and illumination with white LED ring light cover to protect lens. The camera communicates via an Ethernet connection and supports protocols such as Ethernet / IP, FTP, PROFINET, OPC, Modbus / TCP, TCP / IP, SLMP and RS-232. As the COGNEX is7802 camera we use also supports the FTP protocol, we decided to use an FTP server created on a computer with the Windows 10 operating system to transfer data [8].

\section{A. Create an FTP server}

We decided to create an FTP server on a computer that is on the same network as the camera. Windows 10 supports user and server creation on a single computer. First of all, it is necessary to create a bookmark that will represent the storage space for data. Users will have access to this tab so they can write data to it. The next step is to create an account that will have rights to access the bookmark. This account represents a logged in user with a username and password to access the FTP server. On the path "Control Panel All items of the Control Panel Administrative Tools" we will find a tool called "Internet Information Services (IIS) Manager" and in this tool we will create an FTP page as can be seen in figure 4. 


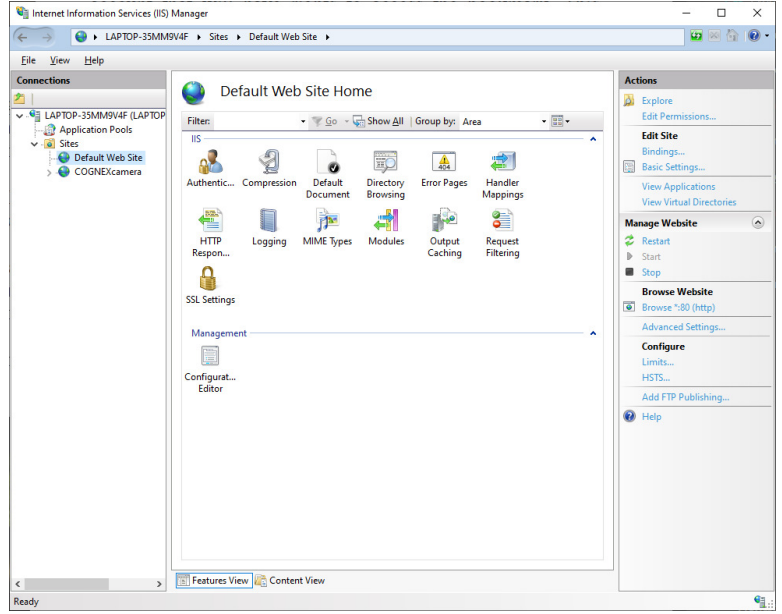

Fig. 4. Creating an FTP Server

Next, in this tool it is necessary to set the path to the shared directory and specify it as a bookmark of the FTP server. In this tool, it is necessary to add your new user and add all the rights (read and write) as can be seen in figure 5 .

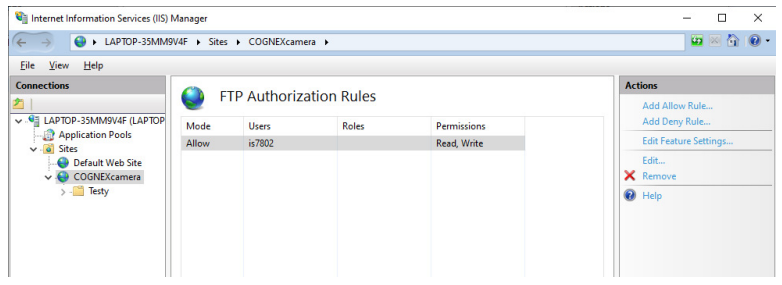

Fig. 5. sers with access to the FTP Server

\section{B. Configure FTP transfer on the camera}

The camera is configured in In-Sight Explorer, which is the software that controls the camera. In this software, all the parameters of the camera are set, and it also determines which image to capture and what parameters to store and process further. In insight explorer, the user has two options for displaying the environment. The first is "EasyBuilder" and the second is "Spreadsheet". EasyBuilder is an intuitive and very friendly graphical display in which the user can create the basic recognition that the camera offers without any knowledge. However, for more complex tasks such as composing multiple functions and then using mathematical operations from the outputs of these functions, it is far better to use the Spreadsheet view. Spreadsheet view offers more features and tools than the EasyBuilder view menu. FTP transfer is feasible in both display options [9].

\section{Konfiguration in EasyBuilder view}

When configured in the middle, the setup is relatively simple. In the panel that offers application steps, select the "Communication" option and in the lower left corner you will see the types of possible connections, including FTP. The window with application steps can be seen in figure 6 .

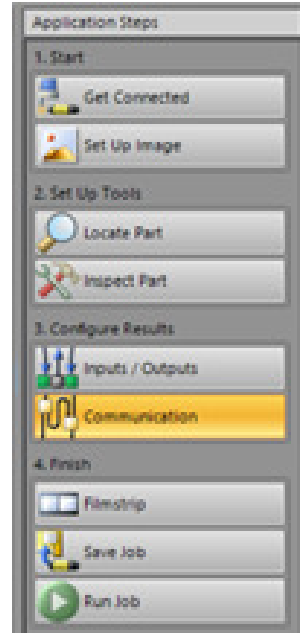

Fig. 6. Application steps

Then assign Host Name in the communication settings, which is the IP address and port of the FTP server in the format "IPaddress:Port". It is necessary to set a different FTP server port than the camera itself. Next, fill in the User Name and Password, these are the data of the user you created. You can see the connection settings in Figure 7.

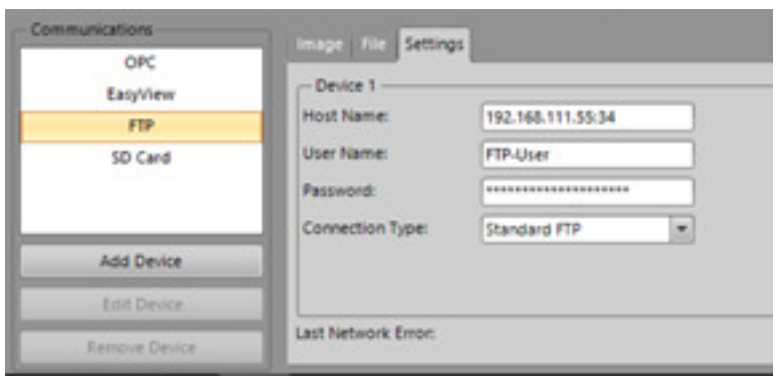

Fig. 7. Connection settings in EasyBuilder

\section{Konfiguration in Spreadsheet view}

The spreadsheet view represents a cellular environment similar to Microsoft Office Excel. It is possible to insert functions or function outputs into these cells for further possible data processing. Functions ensuring FTP data transfer can be found in the Input / Output; Network category. Figure 8 shows the position of the palette with functions in right side and the cell editor in left side.

When configured, the WriteFTP or WriteImageFTP function has the write format:

\section{WriteFTP(Event, HostName, User Name, Password, FileName, DataFormat, String, Append)

On figure 9 you can see example how are written data chosen by us only for one type of testing process from camera to $\mathrm{PC}$. 


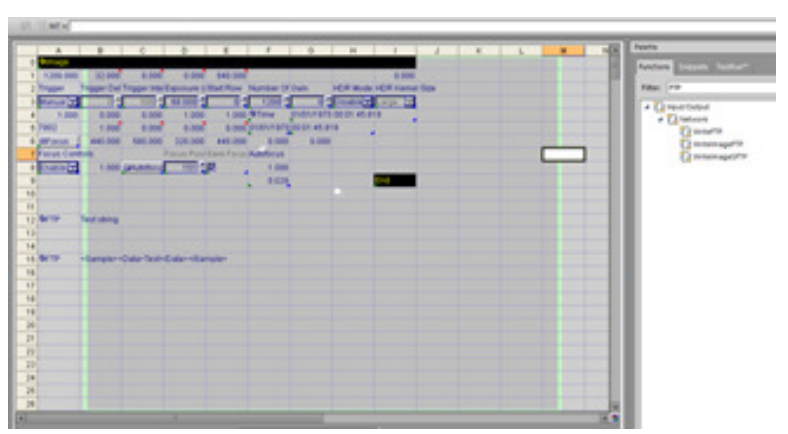

Fig. 8. Spreadsheet view

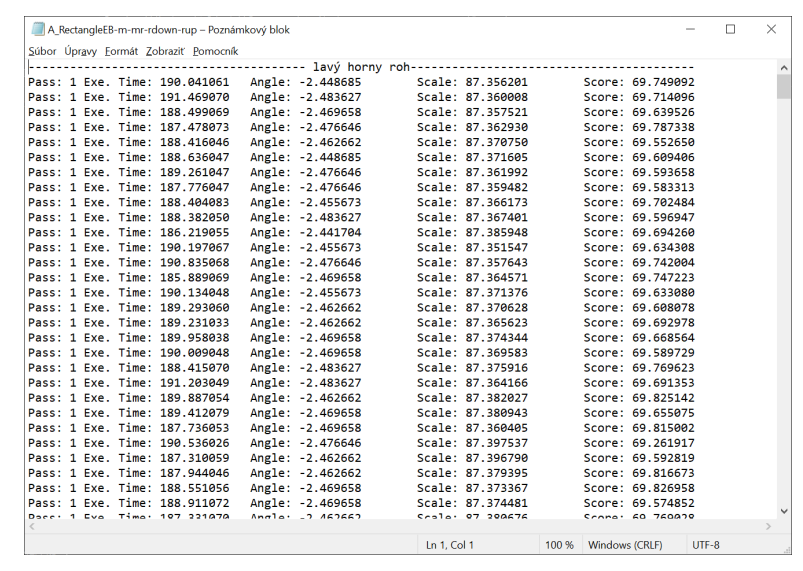

Fig. 9. Written Data

\section{COMPARISON OF RECOGNITION SPEEDS OF BASIC GEOMETRIC SHAPES}

First, it was necessary to find out which geometric shape is most easily recognizable. During the testing, we tested 3 geometric shapes, namely a rectangle, a circle and a triangle. The test was carried out. Thus, these geometric shapes were each printed with a size of $15 \mathrm{~cm}$ and a side thickness of 1 $\mathrm{cm}$.

These faces were attached to a solid surface with which it moved in front of the camera at a distance of $2.5 \mathrm{~m}$. The purpose of these tests was to find out which department recognizes the shortest and also to find the advantages and disadvantages of use for each department. We decided to perform 3 experiments for these units. The first attempt was focused on recognizing features in different positions in front of the camera. These positions were distributed throughout the camera's field of view. The second attempt was focused on the percentage overlap of the pattern when image can still be recognized. The 3rd experiment was designed to determine how the recognition time will affect the rotation of the pattern in front of the camera.

These experiments are based on parameters that can be set on the camera we use. These parameters include Image Distortion, for example, if the lens uses the Fisheye Effect. The first test was also focused on this. Another parameter is the angle below which the image must recognize, and therefore for each shape it is necessary to set the angle according to the sides of the geometric shape.

\section{A. Pattern Position in Camera View}

In front of the camera, we measured the positions where we will place the objects. There were 9 of these possible object fields. You can see them in figure 10 as smaller rectangles with position indicators. Camera view is represented by bigger full rectangle border. This camera view is picture which can be captured by camera.

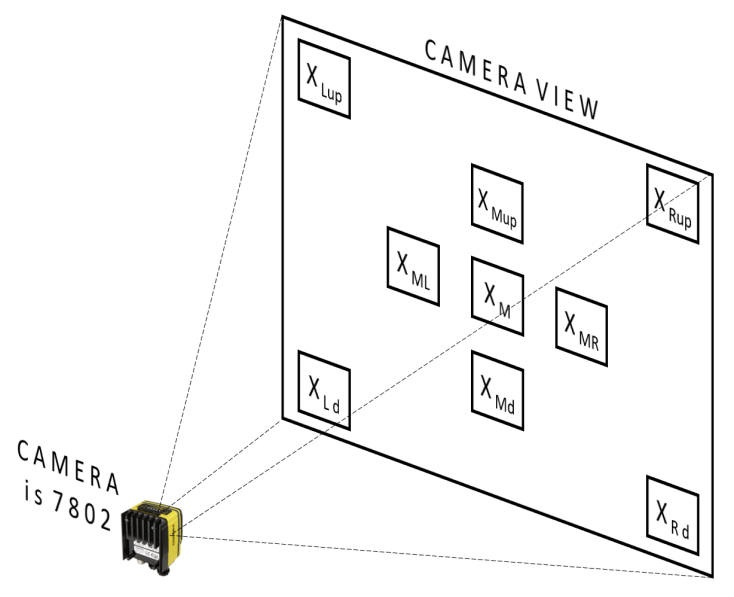

Fig. 10. Camera View

In InSight Explorer, we created a script to train and learn to further recognize a geometric shape if it appears in front of the camera and if it recognizes time information, how long it took to discover other information, such as resizing an image or how many percent it matches the trained one. picture. In this test, the decisive time for which the image was discovered and also how much it was necessary to increase the image alignment parameter to be recognized in the corners of lenses where the image is distorted.

The calibrated image or thus the trained image was at the exact center and the parameters were further adjusted so that this image was recognizable to other parts of the screen. This way we have achieved the accuracy that at any place where the camera captures the image and this image will be recognized. In this test, 100 separate measurements were performed in each position on 100 different images together, so there were 900 measurements for which we calculated the average recognition time and stopped the Graph at which the position took longer and at which the time took shorter.

In figures 11 and 12, you can see graphs that plot the recognition time and the score at which the image was recognized. The score above represents the percentage of how much the pattern resembles the learned pattern. The $\mathrm{x}$-axis represents the time course of 100 consecutive images.

\section{B. Percentage overlap of the pattern}

In the second test, we wanted to find out what percentage of geometric shapes can be overlapped and still be recognized 

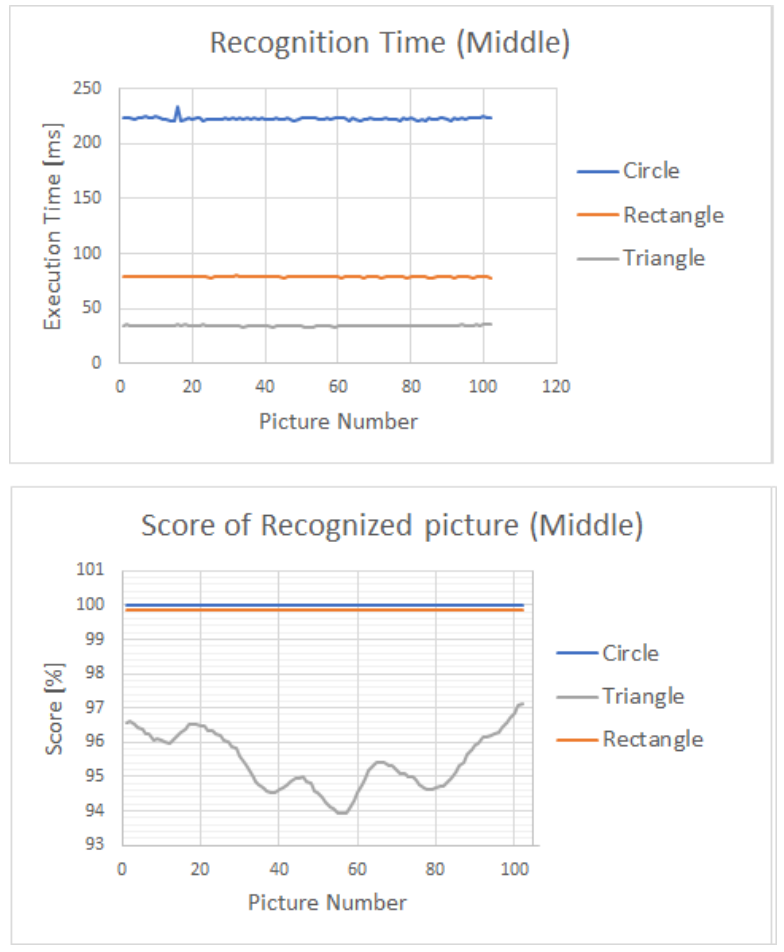

Fig. 11. Graphs of Recognition in the middle of camera view
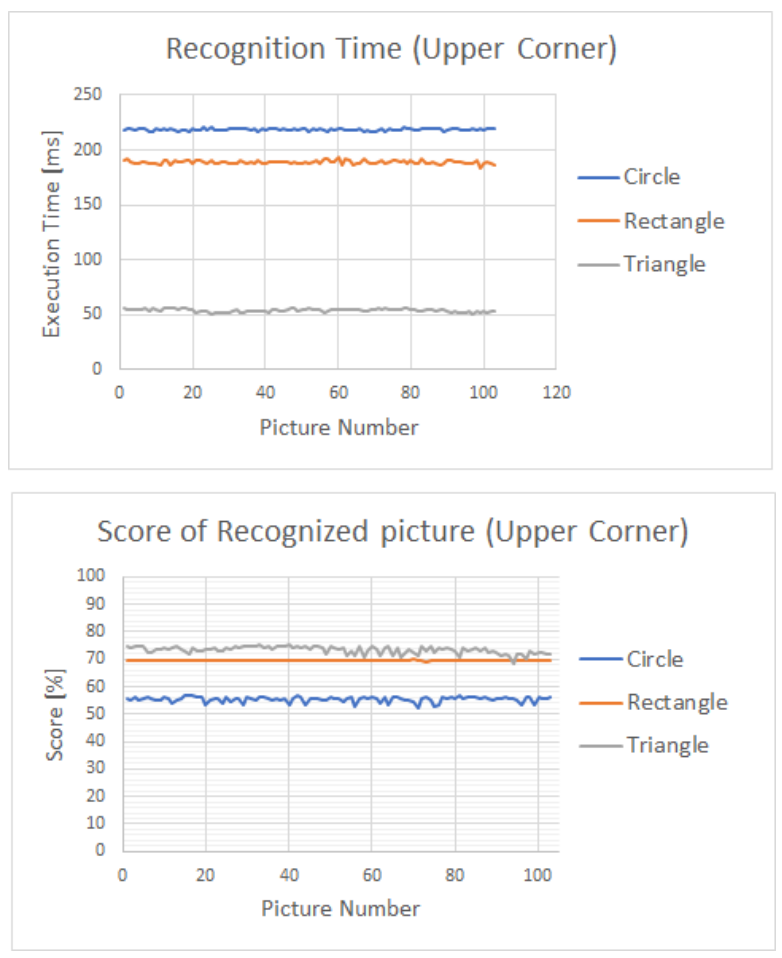

Fig. 12. Graphs of Recognition in the Upper Corners of camera view by the camera. This test was intended to assume that these signs would be placed on the road. And so there is a risk that they may be partially damaged or covered or soiled. In this test, the pattern was calibrated exactly to the centre of the camera. Other parameters such as rotation or percentage enlargement or reduction of the image were set to zero so that it was purely detectable without any other parameters. This percentage is represented by a variable called a score. You can see in the graphs in figure 13 the minimum value for the coverage the system was still able to recognize the pattern.

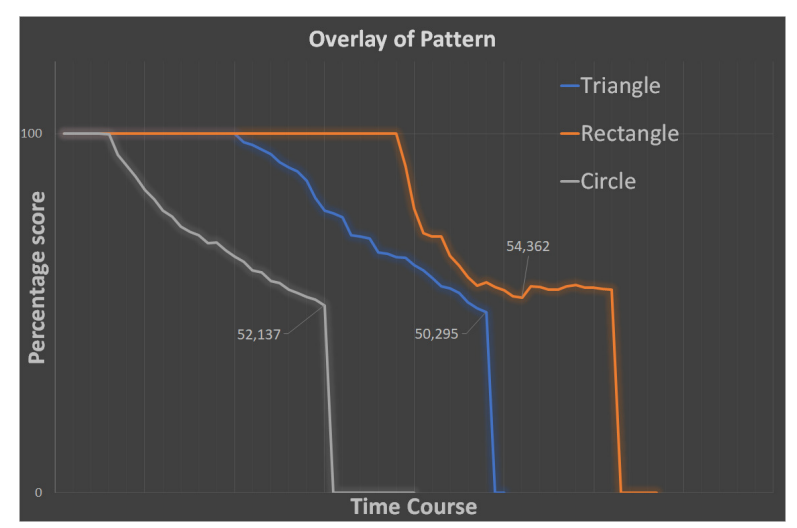

Fig. 13. Graph of Overlay of Pattern

This test was performed so that the pattern with the pattern gradually overlapped from each Cardinal directions and their combination, that is, there were 8 tests for each pattern, which were averaged, and the last data in the variable score was the lowest value at which the camera can recognize the pattern.

\section{Rotation in Front of the Camera}

Another test was to determine the time needed to recognize the pattern if it rotates with it. This test was to determine how the angle and rotation of the pattern would affect the recognition time. This text was converted so that for each image you set the angle needed for $100 \%$ image recognition. For a circle it was $0^{\circ}$, for a rectangle it was $90^{\circ}$ and for a triangle this setting was $120^{\circ}$. These settings are set in InSight Explorer, which provides camera control. In this test, two hundred images were used for each pattern. Each of these images was different and the output from the camera recognition was to find out how the camera system perceives the change of angle for further image processing. You can see the results of these tests in the following graph in figure 14 .

As can be seen in Figure 14, the camera is absolutely unable to detect the rotation of the pattern. In this test, the graph should rotate to form a sinusoid at the square and triangle and at the circle will be straight. However, the camera cannot work with the same side, for example if we imagine a square with sides a1, a2, a3 and a4 with a1 being down and a3 up in a horizontal position and we rotate the pattern 90 or 180 degrees etc. so for the camera it still represents from the pattern is rotated by 0 degrees. The same goes for the triangle just that there is an angle of 120 degrees. 


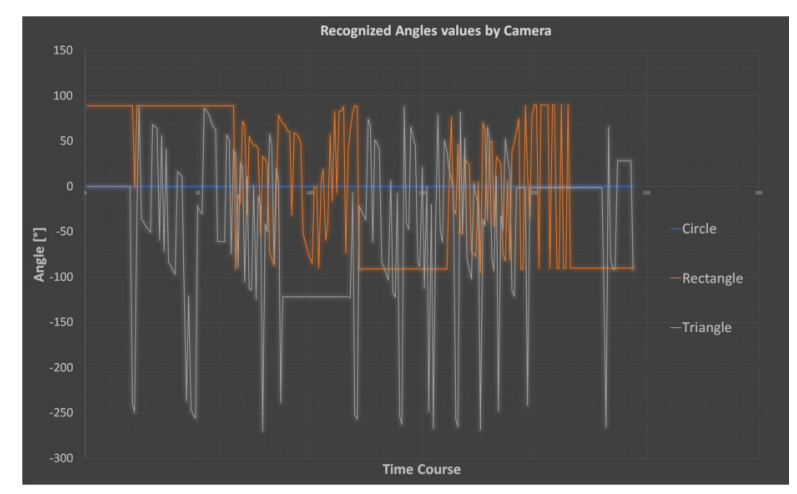

Fig. 14. Recognized Angles values by Camera

\section{DESIGN OF R-ID}

After the tests, we decided to use the triangle as the most ideal shape for recognition by our camera. However, it still needs to be modified so that it can provide information about the rotation of the vehicle. It should also be borne in mind that the shape will be painted on the road and therefore the substrate must consist of one piece. Therefore, we adjusted the shape to the form as can be seen in figure 15 .

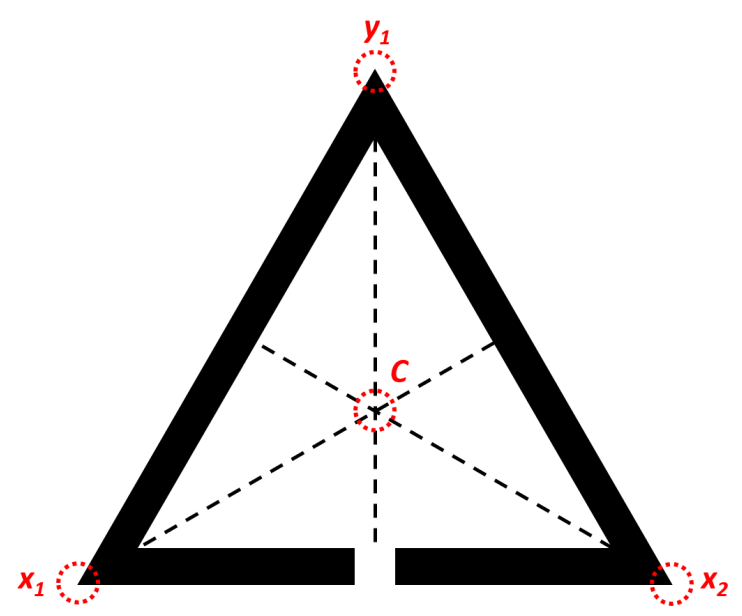

Fig. 15. Simple R-ID

With these changes we will provide a reference point $\mathrm{C}$ which will be accurately measured by surveyors. It will also provide us with points $y_{1}$ and $x_{1}$ and $x_{2}$, which will be subject to further measurements and measurement accuracy in order to determine the distance between the camera mounted on the vehicle and the road identifier.

\section{FUTURE WORK}

Since in this research we found that the triangular shape is the most ideal for use as a road identifier. It is necessary to test this the upper corner of the triangle and in the middle of the opposite side of this triangle.

The centre of the triangle is the intersection of such 3 sections of lines that begin at the vertex and end at the centre of the opposite side of the vertex. At this intersection will be the calibrated position using a static satellite module which will be accurately measured by the surveyor and its accuracy should be a maximum of a few tens of millimetres. With the help of thousands of tests, this static GPS module can determine a very precise position, which cannot be determined with high accuracy when the vehicle is moving in enclosed covered areas. Thus, this pattern will represent an exact imaginary milestone that will determine the exact position of the vehicle and also the direction in which the vehicle came to the image.

Other plans include the fusion of several sensors and systems and technologies, such as a camera with a new network that will ensure safety in front of the vehicle by having trained algorithms and neural networks to recognize objects of persons or other obstacles that unexpectedly appear in front of the vehicle. The other data that will be merged will come from the Vehicle Odometry and this data will talk about the speed of the expected time to the next identifier of the rotation of the vehicle's wheels and the tracking of the trajectory through which the vehicle has passed. The vehicle will also contain a GPS module, which does not assume high accuracy, but will be a security element that will specify the camera system whose purpose is to recognize the pattern on the ground. It is also possible to use systems such as LIDAR when mounted on servomotors and ultrasonic sensors around the perimeter of the vehicle from each side.

\section{CONCLUSION}

The article describes the decision-making procedure for the design of an R-ID using camera recognition of geometric shapes. Important parameters are e.g. the uniqueness of the R-ID and the percentage needed for the R-ID to be correctly recognized by the camera and to obtain the necessary data from the overlay image. However, the most important parameters to be found in the shortest possible time are the most accurate position of the vehicle and the direction from which it comes to this position; they are still to be determined.

\begin{tabular}{c|c|} 
Pattern & $\begin{array}{c}\text { Average time of } \\
\text { recognition pattern [ms] }\end{array}$ \\
\hline Circle & 72,715 \\
\hline Triangle & 80,576 \\
\hline Rectangle & 87,685 \\
\hline
\end{tabular}

Fig. 16. Execution Times of Overlay test

\begin{tabular}{c|c|} 
Pattern & $\begin{array}{c}\text { Average time of } \\
\text { recognition pattern [ms] }\end{array}$ \\
\hline Circle & 64,427 \\
\hline Triangle & 138,068 \\
\hline Rectangle & 122,263 \\
\hline
\end{tabular}

Fig. 17. Execution Times of Rotation test

As can be seen from the tables (figures 16,17 and 18), the largest differences in recognition were measured in tests when there were maximum recognition requirements, i.e. it 


\begin{tabular}{|r|c|c|c|c|c|} 
& \multicolumn{5}{|c|}{ Average Recognition Time [ms] } \\
\hline Patterns & $\begin{array}{c}\text { Upper } \\
\text { Corners }\end{array}$ & $\begin{array}{c}\text { Lower } \\
\text { Corners }\end{array}$ & Centre & $\begin{array}{c}\text { Centre } \\
\text { Up / Down }\end{array}$ & $\begin{array}{c}\text { Centre } \\
\text { L/R }\end{array}$ \\
\hline Triangle & 53,928 & 51,203 & 34,406 & 32,413 & 38,191 \\
\hline Rectangle & 188,914 & 240,710 & 78,712 & 78,252 & 76,750 \\
\hline Circle & 218,484 & 219,412 & 222,980 & 222,162 & 224,081 \\
\hline
\end{tabular}

Fig. 18. Execution Times of Position test

was necessary to recognize a pattern in any part of the scanned image. When rotating the image, the triangle ended with the time in the last place, but in real operation it is not possible for the vehicle to approach the image painted on the road at an angle greater than 120 degrees, so this result should not be considered too important. In further tests, a lower range of possible angles will be assumed. When testing the overlap of the pattern, the times were relatively the same, but it should be noted that when recognizing overlapping patterns, the best percentage of the right triangle was 50.295 (meaning that $49.705 \%$ of the image can be obscured from the pattern), 52.573 which is an overlap of $47.827 \%$ and with a rectangle score of 54.362 is an overlap of $45.638 \%$. Therefore, we decided to use a triangle as R-ID.

Camera limitations consist mainly in a poor resolution. On the resolution depends the calibration and the conversion of pixels to millimeters required when measuring the position. During testing, the camera was not mounted on the vehicle but was located only on a tripod that represented a model of a stationary vehicle. This means that it was mounted at the same height and under the same inclination as will be mounted on the vehicle, and the tested patterns were placed in similar distances as they will be painted on the road. After transferring the information from the camera to the server, the computer selects the information from the server and further processes it. The computer's task will be to calculate the distance and rotation from the data ( $\mathrm{x}$ and $\mathrm{y}$ values) received from the camera. This data is then compared with the data from other sensors according to the time stamp. When the computer knows the exact position of the vehicle, it sends a direction correction so that the vehicle is directed to the next mark. The aim of road identifiers is not navigate the autonomous vehicle but to specify its position in places where other sensors are less reliable, and in the end to assist in the navigation of the autonomous vehicle.

\section{ACKNOWLEDGMENT}

The paper was partially supported by the Slovak Research and Development Agency, grant No. APVV-17-0190 and the Slovak Cultural Educational Grant Agency, grant No.038STU$4 / 2018$.

\section{REFERENCES}

[1] F. Valocký, P. Drahoš, and O. Haffner, "Measure distance between camera and object using camera sensor," in 30th Conference Cybernetics \& Informatics. FEI STU BA, 2020.

[2] B. Buková, R. Madleňák, and K. R., "Elektronické podnikanie v doprave a logistike." Iura Edition, 2009.

[3] Opel, Groupe PSA, "Innovative Opel Eye camera," 2014. [Online] Available: https://http://www.opel.pl

[4] T. Corejová, A. Križanová, and N. A., "Tendencies in the professional education for transportation sector in slovakia." Iura Edition, 2009.

[5] J. Mikulski and P. Czech, "Vibration signals to diagnose damage of head gasket in internal combustion engine of a car. in: Telematics." TST 2014, 2014.

[6] VolvoCars, "NightVision," 2014. [Online]. Available: http://www. volvocars.pl/september2014

[7] R. Fujdiak, P. Masek, P. Mlynek, J. Misurec, and A. Muthanna, "Advanced optimization method for improving the urban traffic management," in Proceedings of the 18th Conference of Open Innovations Association FRUCT. FRUCT Oy, 2016, pp. 48-53.

[8] COGNEX, "Cognex Support," 2019. [Online]. Available: [6]https://support.cognex.com/docs/is_580/web/EN/ise/Content/ Reference/WriteFTP.htm?tocpath=Communications\%20Reference\% 7CFTP\%20Communications\%7C

[9] F. Valocký, P. Drahoš, O. Haffner, and M. Orgoň, "Transfer data from camera required for autonomous driving via ftp," in 22nd Conference of Doctoral Students Faculty o fElectrical Engineering and Information Technology. FEI STU BA, 2020. 